\title{
On the Validity Extent of Linear Viscoelastic Models of Human Brain
}

\author{
Hesam Hoursan \\ Ph.D Student, School of Mechanical Engineering \\ Sharif University of Technology \\ Tehran, Iran \\ $\underline{\text { Hesam.hoursan74@student.sharif.edu }}$
}

\author{
Reyhaneh Kazemiasfeh \\ Taleghani Hospital \& Medical Center \\ Tehran, Iran \\ rkazemi68@gmail.com
}

\author{
Mohammad Taghi Ahmadian \\ Professor, School of Mechanical Engineering \\ Sharif University of Technology \\ Tehran, Iran \\ Ahmadian@sharif.edu
}

\author{
Ahmad Barari \\ Faculty of Engineering and Applied Science \\ University of Ontario Institute of Technology \\ Oshawa, Ontario, Canada \\ Ahmad.barari@uoit.ca
}

\begin{abstract}
Characterization of human brain material properties in the form of computationally feasible mathematical models is a complex problem; especially when the models are used in complicated Finite Element simulations. Various models have been proposed to include the tissue's hyper-viscoelasticity, most of which are quite complicated and therefore only suited to Software-based Finite Element methods. Use of linear material models simplifies the problem and saves much time and effort, allowing the researcher to verify the results of more sophisticated models with lower computational cost. However, the preciseness of the results from such models is subject to special conditions. This study proposes and validates a Generalized Maxwell linear viscoelastic model with five constants to be used as an acceptable computational method to simulate brain's viscoelastic behavior at low strain rates. To this end, an explicit numerical integration scheme is used to simulate the single-DOF tissue response with a Generalized Maxwell viscoelastic model. Using the material constants of a previous hyper-viscoelastic model, the results are compared with those obtained from a previous experiment. The comparison shows that the linear GM viscoelastic model is predicting the low-strain-rate behavior of the brain tissue with acceptable error.
\end{abstract}

Keywords- Viscoelasticity; Finite Element; Generalized Maxwell, Linear Viscoelastic Model; Strain Rate

\section{1- INTRODUCTION}

The head is often considered as the most critical region of the human body for life-threatening injuries as result of accidents. The cost incurred by the accidents is estimated to be 160 billion Euros per year in the European Union alone [1]. In a 6-year period, an average of 1.4 million cases of traumatic brain injury occurred in the United States each year, of which $20 \%$ resulted from motor vehicles accidents. In order to develop effective protective measures, a better understanding of the process of injury development in the brain is required. [2-5]

Over the past three decades, several researchers have investigated the mechanical properties of brain tissue in order to establish constitutive relationships over a wide range of loading conditions. In experimental methods, dynamic oscillatory shear tests $[4,8,9]$ and unconfined compression tests [10] have been conducted more frequently. The resulting constitutive models have been introduced in Finite Element (FE) analyses to simulate the actual response of the tissue to external stimuli. Among these modes, most of them use hyperviscoelastic constitutive material assumption which, when combined with the complex geometry of brain parts, lead to complicated analyses [11]. The nonlinearity included in such models may lead to distorted results if the convergence problem is not handled. Therefore, simpler linear models such as the one presented here can serve as useful tools for verification of these models.

In current FE head models, brain tissue is commonly assumed to display hyper-viscoelastic material behavior. The tissue behaves like a non-linear viscoelastic solid for shear strains above $1 \%$. The modelling of the tissue behavior with these assumptions leads to somewhat more accurate results than linear models. However, it maintains the big drawback of high 
computational cost and time-consuming nature of these constitutive nonlinear models. [5, 6, 12]. Simpler computational frameworks such as the one presented herein serve as useful tools for engineering approximations of the resulting values of stress and strain fields.

This study seeks to investigate the extent of validity of a linear viscoelastic numerical model for modelling the response of the brain tissue to single-DOF loading-unloading cycles of shear strain. In order to evaluate the model, the results are compared, on various levels, with those of an experimental study.

\section{2- METHODOLOGY}

\section{2-1- Derivation of Mathematical Models}

\section{Generalized Maxwell Model}

In order to derive a computational framework for solving the Generalized Maxwell (GM) model (figure 1), one may derive the equation for a single mode spring and damper in series and then sum over the number of modes. The effect of infinity spring is finally added to complete the model.

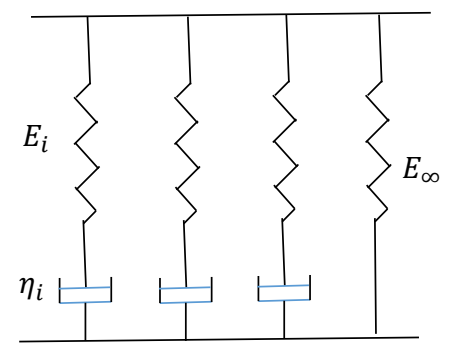

Figure 1- The Generalized Maxwell (GM) model for viscoelastic materials. $E_{i}$ and $\eta_{i}$ are serial spring and damping constants and $E_{\infty}$ is the parallel spring constant

Considering a single mode spring and damper in series and the infinity spring in parallel, the stress can be written in terms of strain and strain rate in the following form,

$\sigma(t)=E_{\infty} \epsilon(t)+\sigma^{*}$

where

$\sigma^{*}=\boldsymbol{\eta} \frac{\mathrm{d} \epsilon^{*}}{\mathrm{dt}}$

Solving and integrating the equation above for $\epsilon(t)$, the integral equation for two consecutive steps is obtained in the following form,

$\sigma_{n+1}^{*}-\sigma_{n}^{*}=\frac{-1}{\lambda} \int_{t_{n}}^{t_{n+1}} \sigma^{*}(t) d t+E\left(\epsilon_{n+1}^{*}-\epsilon_{n}^{*}\right)$

Considering that the integral is from $t_{n}$ to $t_{n+1}$, it's possible to approximate it using Implicit or Explicit method (Forward Euler and Backward Euler)
Substituting,

$\sigma^{*}(t)=E \epsilon(t)-y(t)$

Where,

$\frac{d y(t)}{d t}=\frac{-1}{\lambda} y(t)+\frac{E}{\lambda} \epsilon(t)$

An explicit numerical method can be obtained to approximate the integral in the following form:

$$
\sigma_{n+1}^{*}=e^{\frac{-\Delta t}{\lambda}} \sigma_{n}^{*}+e^{\frac{-\Delta t}{\lambda}} E \Delta \epsilon_{n}
$$

Now one can add the effects of all the parallel units the single-mode model. $\sigma_{n}^{*}$ is calculated for each unit and finally summed to obtain the total $\sigma^{*}$.

$$
\sigma^{*}=\sigma_{1}^{*}+\sigma_{2}^{*}+\cdots+\sigma_{n}^{*}
$$

The total $\sigma^{*}$ is subsequently added to the effect of infinity spring to yield the total stress by substituting into equation 1 . In the above equations, $\mathrm{n}$ is the order of GM model denoting the number of parallel modes. Time constants need to be calculated for every parallel Maxwell unit and considered separately in the corresponding stress update algorithm:

$$
\lambda_{i}=\frac{\eta_{i}}{E_{i}}
$$

\section{2-2- Application of Load}

Two load scenarios were considered as input, namely Load Scenario A and Load Scenario B. The load scenarios were chosen to replicate a previous experiment on samples from human head $(\operatorname{Ref}[4])$

\section{2-2-1- Load Scenario A}

In this load scenario, triangular pulses with constant strain rate of 1.5 /s were applied to the viscoelastic single-dimensional model. The input pulses were obtained from [4] and simulated as triangular pulses (Fig. 2). The strain was applied as input by using explicit finite difference method with 600 steps and time constant of 1.1s. The strain rate was kept constant at the value of $1.5 / \mathrm{s}$ (Table 1$)$. 


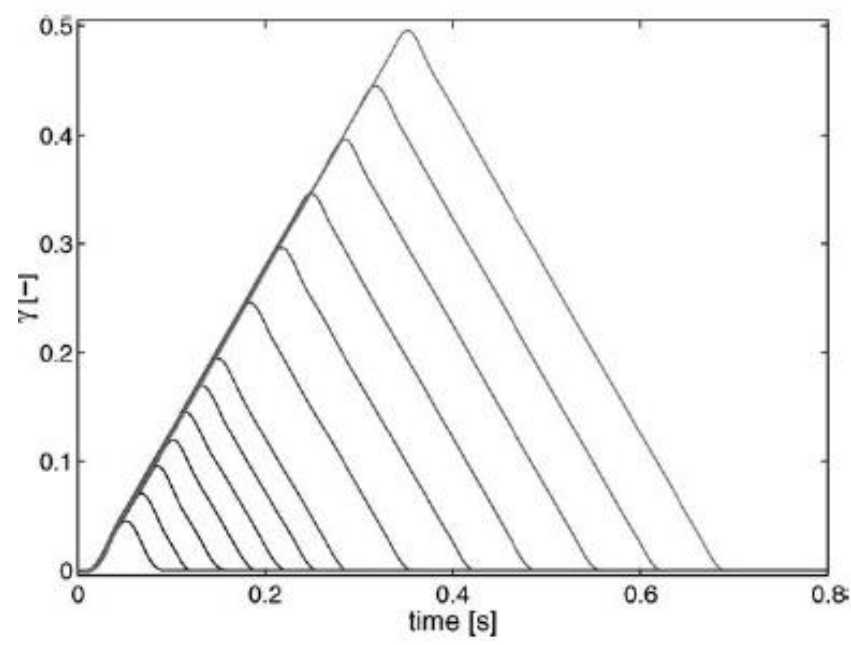

Figure 2- Triangular pulses (constant shear rate in loading and unloading) of Scenario A [4]

Table 1- Characteristics of the Triangular pulses of Scenario A [4]

\begin{tabular}{|c|c|c|c|}
\hline $\begin{array}{c}\text { Pulse } \\
\text { number }\end{array}$ & $\begin{array}{c}\text { Max. } \\
\text { Strain }\end{array}$ & $\begin{array}{c}\text { Pulse } \\
\text { Duration (s) }\end{array}$ & $\begin{array}{c}\text { strain Rate } \\
(/ \mathbf{s})\end{array}$ \\
\hline $\mathbf{1}$ & 0.05 & 0.06 & 1.50 \\
\hline $\mathbf{2}$ & 0.10 & 0.13 & 1.50 \\
\hline $\mathbf{3}$ & 0.15 & 0.20 & 1.50 \\
\hline $\mathbf{4}$ & 0.20 & 0.26 & 1.50 \\
\hline $\mathbf{5}$ & 0.30 & 0.40 & 1.50 \\
\hline $\mathbf{6}$ & 0.50 & 0.66 & 1.50 \\
\hline
\end{tabular}

\section{2-2-2- Load Scenario B}

In this load scenario, triangular pulses with various strain rates of $1 / \mathrm{s}, 0.1 / \mathrm{s}$ and $0.01 / \mathrm{s}$ were applied to the viscoelastic single-dimensional model. Table 2 shows the characteristics of the input strain pulses applied to the computational model.

Table 2- Characteristics of the Triangular pulses of Scenario B

\begin{tabular}{|c|c|c|c|}
\hline $\begin{array}{c}\text { Pulse } \\
\text { number }\end{array}$ & $\begin{array}{c}\text { Max. } \\
\text { Strain }\end{array}$ & $\begin{array}{c}\text { Pulse end } \\
\text { time (s) }\end{array}$ & $\begin{array}{c}\text { Strain rate } \\
(/ \mathbf{s})\end{array}$ \\
\hline $\mathbf{1}$ & 0.1 & 0.2000 & 1 \\
\hline $\mathbf{2}$ & 0.1 & 2.0000 & 0.1 \\
\hline $\mathbf{3}$ & 0.1 & 20.0000 & 0.01 \\
\hline
\end{tabular}

\section{3- Material constants}

For the 5-branch GM linear viscoelastic model, 5 sets of material data including the instantaneous and equilibrium shear moduli, time constant, and viscous damping coefficient was obtained from Ref [4]. The input data was included in the GM model in the form of Prony Series (Table 3)

Table 3-constants of the GM model obtained from curve-fitting of a hyperviscoelastic model on the experimental results of Ref [4]

\begin{tabular}{|c|c|c|c|c|}
\hline $\begin{array}{c}\text { GM } \\
\text { Mode } \\
\text { Number }\end{array}$ & $\begin{array}{c}\text { G } \\
\text { (Modulus) } \\
\text { (Pa) }\end{array}$ & $\begin{array}{c}\boldsymbol{\tau} \text { (time } \\
\text { constant) } \\
\text { (S) }\end{array}$ & $\begin{array}{c}\text { ๆ (Viscous } \\
\text { damping } \\
\text { coefficient) }\end{array}$ & $\begin{array}{c}\text { Ge } \\
\text { (Equilibrium } \\
\text { Modulus) }\end{array}$ \\
\hline Mode 1 & 835.50 & 0.012 & 10.02 & 182.90 \\
\hline Mode 2 & 231.20 & 0.35 & 80.92 & 182.90 \\
\hline Mode 3 & 67.10 & 4.62 & 310.00 & 182.90 \\
\hline Mode 4 & 3.610 & 12.13 & 43.68 & 182.90 \\
\hline Mode 5 & 2.79 & 54.31 & 151.49 & 182.90 \\
\hline
\end{tabular}

\section{4- Results}

The results of the application of nine strain pulses from two scenarios to the GM model are shown in figures 3 and 4. Fig. 3 indicates the resulting stress from input strain of load scenario A with constant strain rate, and Fig. 4 shows the effect of changing the strain rate (load scenario B)

In all calculations, in order to reach better convergence and considering a recovery time as two times a full pulse, explicit algorithm has been used with 600 steps. 


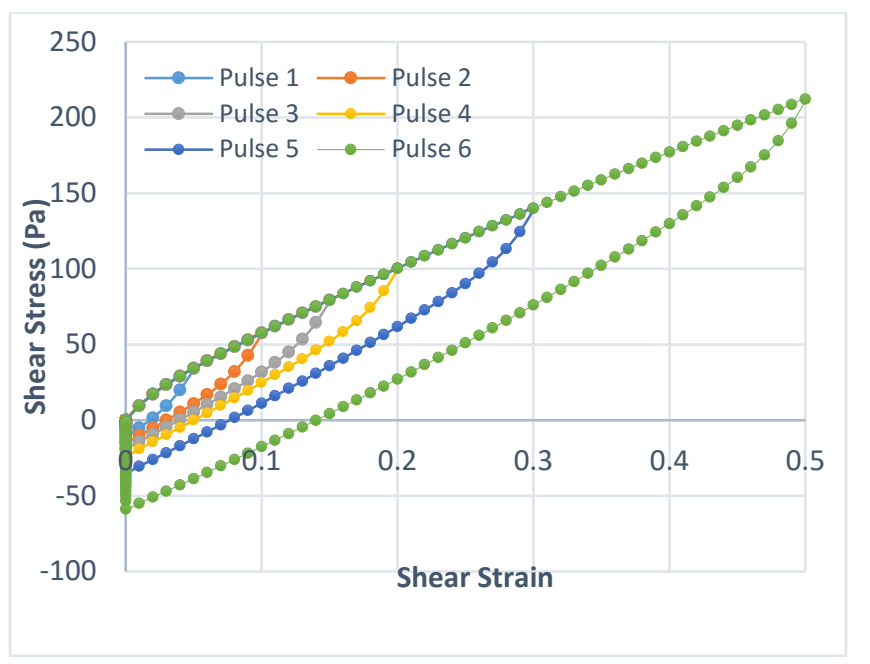

Figure 3 -Stress-Strain results obtained from applying the input pulses of Scenario A to the Generalized Maxwell Model (5 branches)

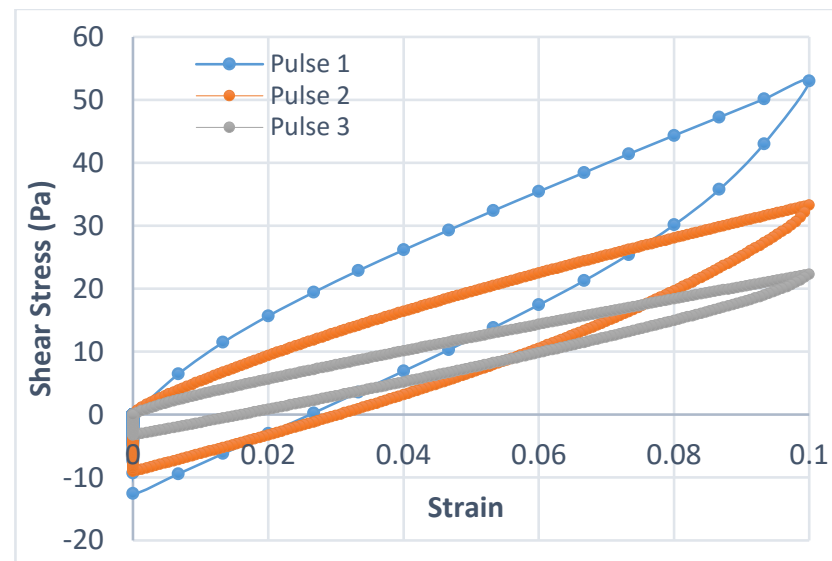

Figure 4 -Stress-Strain results obtained from applying the input pulses of Scenario B to the Generalized Maxwell Model (5 branches)

\section{4- Discussion of Results}

Nine input strain pulses were applied to a single-DOF linear GM viscoelastic model in two load scenarios. The resulting stress was calculated by using a finite difference integration scheme and plotted against the stress for all pulses (Fig. 3, 4). The input strain and material constants were obtained from curve-fitting of a hyper-viscoelastic model on a set of experimental curves from the literature [4].

The results from the application of the same load pulses in the referenced experiment are shown in Fig 5 and 6. As it can be observed, the Generalized Maxwell of order 5 shows acceptable qualitative agreement with those of the experiment. As it was predicted, the similarity of the trends is more noticeable at lower strain rates. The relative error at the end of the loading cycle (i.e. at maximum strain) for pulse 3 is $3 \%$ with respect to the experimental value. This can be observed by comparison of Fig. 3 and 5. The linearity of the model leads to differences with the experimental results which are more pronounced at higher input strains and strain rates. At the strain of 0.5 , the result of stress shows $10 \%$ deviation from the experimental results (pulse 6 from load scenario A). In addition, the quantitative comparison of the results reveals the incapability of the linear model in prediction of the material behavior at high strain rates and high strains (by comparison of the stress in pulse 1 from scenario B and the corresponding curve in Fig. 6). At the strain rate of $0.1 / \mathrm{s}$ and strain of 0.1 , the relative error of the linear model with respect to experiment results is $33 \%$ which is more than the one reported by the hyper-viscoelastic model of reference [4] $(21 \%)$.

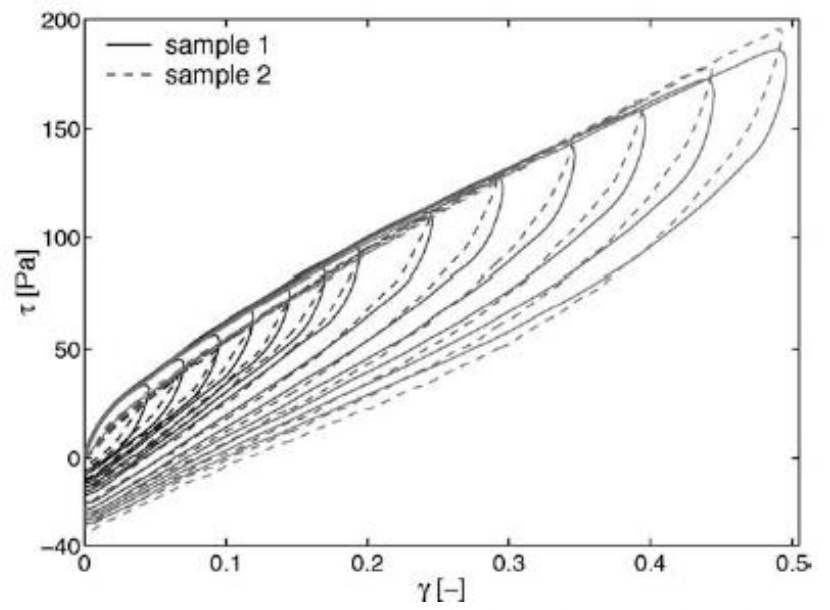

Figure 5-Stress-Strain curve (Curve-fitted) obtained from applying the input pulses of Scenario A (constant shear rate) in the experiment of Ref [4] for two samples.

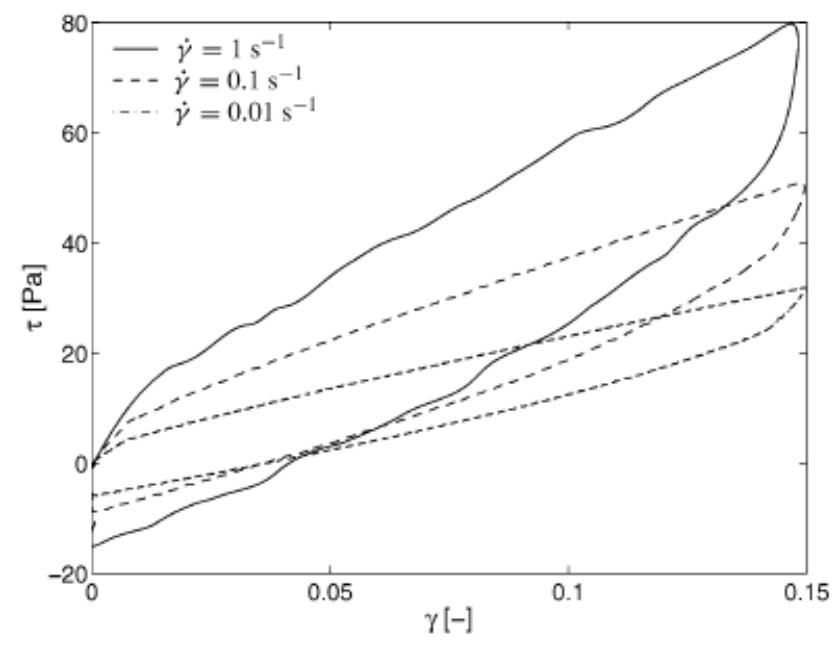

Figure 6-Stress-Strain curve obtained from applying the input pulses of Scenario B (constant shear rate) in the experiment of Ref [4] 


\section{5- Conclusions}

In this study, a linear viscoelastic model was used to assess the capability of linear computational models to model the complex behavior of brain material. 9 input strain pulses were replicated from a previous experiment and applied to the model and the resulting stress was compared with the ones reported in the literature.

It was observed that the results of our model show good qualitative agreement with those of the experiment. In terms of quantity, however, the agreement of the results is restricted to lower strain values and strain rates. Also, the impact of strain rate on the difference seems to be more noticeable than the absolute value of strain input. However, it seems that the mentioned deviation does not justify using a nonlinear hyperviscoelastic model in some studies instead of a linear viscoelastic one which is simpler and has a lower computational cost. It should also be noted that, in order to use the linear viscoelastic model, the parameters of the model must be obtained through direct comparison with experimental results, which may lead to improvements in the accuracy of the present model.

Finally, the comparison of the results reveals that the linear model, although not capable to fully trace the hyper-viscoelastic nature of the phenomena, displays a maximum relative error of $10 \%$, which can be justified given the lower computational cost. This can especially be useful in real-time simulations of surgical procedures, tumor growth and other applications involving change in brain tissue with low strain rates. The GUI used in this study to simulate and obtain the results can be an example of a useful tool for such applications (Figure 7).

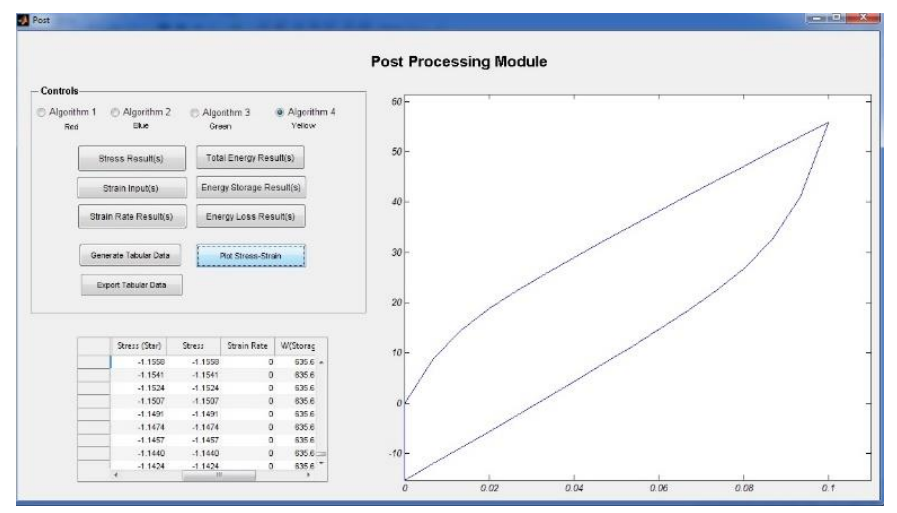

Figure 7- Stress-strain result of the application of pulse No. 2 from Scenario $B$ to the model and visualization of the results via Matlab GUI. The GUI was developed based on the calculations presented in this paper to better visualize the results.

\section{6- References}

[1] J.Ivarsson, D.C,Viano, P.Lovsund, B.Aldman, "Strain relief from the cerebral ventricles during head impact: experimental studies on natural protection of the brain". Journal of Biomechanics 2000, No: 33, pp.181189.

[2] S.S.Margulies, L.E.Thibault and T.AGennarelli, "Physical model simulation of brain injury in the primate". J.Biomech 1990, Vol 23, pp. 823-836.

[3] S.S.Margulies and L.E.Thibault. "A proposed tolerance criterion for diffuse axonal injury in man”. J.Biomech 1992, 25 (8) pp: 917-923.

[4] [4] Hrapko M1, van Dommelen JA, Peters GW, Wismans JS., "The mechanical behaviour of brain tissue: large strain response and constitutive modelling." Biorheology. 2006;43(5):623-36.

[5] M.Prange, S.Margulies, "Tissue strain thresholds for axonal injury in the infant brain". Bioengineering conference 2001, BED Vol 50.

[6] W.Hardy, C.D.Foster, M.J.Mason, K.H.Yang and A.I.King, "Investigation of head injury mechanisms using neutral density technology and high speed biplanar xRay", Stapp Car Crash Journal, November 2001, Vol 45, paper no: 2001-22-0016.

[7] S.A.Lippert, M.J.Grimm, "Estimating the material properties of brain tissues at impact frequencies: a curve fitting solution", Summer Bioengineering conference 2003, June 25-29, Sonesta beach resort in key Biscayne, Florida.

[8] K.K. Darvish and J.R. Crandall, Nonlinear viscoelastic effects in oscillatory shear deformation of brain tissue, Med. Eng.\& Phys. 23 (2001), 633-645.

[9] B.R. Donnelly and J. Medige, Shear properties of human brain tissue, J. Biomech. Eng. 119 (1997), 423-432.

[10] M.S. Estes and J.H. McElhaney, Response of brain tissue of compressive loading, in: Proceedings of the 4th ASME J.Biomechanics Conf., 70BHF-13, 1970.

[11] K. Miller, Constitutive modeling of brain tissue: Experiment and theory, J. Biomech. 30 (1997), 1115-1121.

[12] Rashid, Badar \& Destrade, Michel \& Gilchrist, Michael. (2012). Mechanical characterization of brain tissue in tension at dynamic strain rates. Journal of the mechanical behavior of biomedical materials. 33 . 10.1016/j.jmbbm.2012.07.015.

[13] Goriely A, Geers MGD, Holzapfel GA, et al. Mechanics of the brain: perspectives, challenges, and opportunities. Biomechanics and Modeling in Mechanobiology. 2015;14:931-965. doi:10.1007/s10237-015-0662-4. 